\title{
Stellungnahme zum Leserbrief „Die maskierte Gesellschaft“" von Prof. Santiago Ewig, Prof. Sören Gatermann und Prof. Sebastian Lemmen
}

Vieles von dem, was die Autoren des Leserbriefes zum Ausdruck bringen, ist richtig und wichtig und soll deswegen auch nochmals in dieser Antwort Erwähnung finden:

Der Umgang mit den Community-Masken in der Öffentlichkeit ist durchaus verbesserungswürdig. Masken verschmutzen während des Tragens, von daher sollte eine Maske spätestens nach 6 Stunden desinfizierend gereinigt werden. Das bedeutet auch, dass Personen, die z.B. berufsbedingt länger als 6 Stunden eine Maske tragen, eine zweite Maske zur Verfügung haben sollten. Spätestens nach 6 Stunden sollte eine desinfizierende Aufbereitung der Maske erfolgen. Dies kann z. B. durch Waschen bei $>90^{\circ} \mathrm{C}$ in der Waschmaschine oder durch Auskochen der Maske für mindestens 5 Minuten erfolgen. Eine Maschinenwäsche in Niedertemperaturprogrammen sollte aufgrund des erheblichen Kontaminationsrisikos vermieden werden [1]. Nach Gebrauch der Masken sowie nach der Wäsche sollte außerdem auf eine vollständige Trocknung und kontaminationsgeschützte Lagerung geachtet werden. Wird eine Maske zwischenzeitlich abgesetzt, so sollte sie so gelagert werden, dass keine weitere Kontamination erfolgt. Man kann die Maske frei hängend (z.B. auch am Rückspiegel eines PKW) oder in einem geschützten Reservoir (z. B. einer kleinen Plastiktüte, die nach der Verwendung mit heißem Wasser desinfiziert wird) unterbringen. Von der ungeschützten Zwischenlagerung z. B. in einer Hosen- oder Handtasche ist abzuraten.

Wir stimmen mit den Autoren des Leserbriefes auch darin überein, dass Community-Masken keiner Qualitätsnorm unterliegen und in ihrer Effektivität starke Unterschiede aufweisen. Die Gründe hierfür sowie die Forderung nach einer Qualitätsüberprüfung kommerziell hergestellter Masken findet man im Positionspapier [2].
Über den Übertragungsweg des Coronavirus ist in der letzten Zeit viel diskutiert worden. Generell macht es keinen Sinn, das Aerosolspektrum in die Kategorien „Tröpfcheninfektion“ und „aerogene Infektion“ zu unterteilen [3]. Die im menschlichen Körper gebildeten und abgeatmeten Aerosole stellen ein Kontinuum dar. Bereits beim normalen Atmen entstehen Partikel mit einem Spektrum von $50 \mathrm{~nm}$ bis zu $5 \mu \mathrm{m}[4,5]$. Sprechen oder Husten produziert Partikel, die größer sein können als $10 \mu \mathrm{m}$ [6]. Die Tatsache, dass die Intubation zu den Hochrisikoprozeduren z.B. bei SARS-erkrankten Patienten gehörte mit einem relativen Risiko von mehr als 13 [7], spricht für einen Übertragungsweg mit großen Partikeln. Die zuletzt in der Presse beschriebenen Cluster während einer Chorprobe, eines Gottesdienstes oder aber auch der Karnevalsveranstaltung in Gangelt im Kreis Heinsberg sprechen dagegen für eine Infektion über Aerosole, die länger im Schwebezustand bleiben und so größere Distanzen zurücklegen können. Letzterer Hypothese schließen sich mittlerweile zahlreiche Autoren an [3, 8-16]. Das SARS-CoV-2-Virus hat in schwebenden Aerosolen immerhin eine Halbwertszeit von 1,1 Stunden [17]. In Krankenzimmern infizierter Patienten konnten SARS-CoV-2-tragende Partikel nachgewiesen werden, obwohl das Lüftungssystem der Zimmer die Luft alle 6 Minuten vollständig austauschte [18]. Vor diesem Hintergrund lassen sich Menschenmengen in geschlossenen Räumen als primäre Risikokonstellation darstellen. Im Umkehrschluss ist festzustellen und hier liegen die Autoren des Leserbriefes vollkommen richtig, dass Aktivitäten unter freiem Himmel ein wesentlich geringeres Risiko tragen. Mit einer Maske alleine durch den Wald zu laufen ist daher sicher als sinnlos zu betrachten.

Was Belege für die Schutzfunktion der Community-Masken angeht, verweisen wir nochmals auf die ausführliche Diskussion im Positionspapier der DGP [2]. Die im Leserbrief erwähnte Cochrane-Metaanalyse ist eine Neuauflage einer bereits 2011 publizierten Analyse [19], die in ihrer aktuellen Version im Preprint verfügbar ist. Die Analyse beschränkt sich wie jede Cochrane-Analyse auf randomisierte, kontrollierte Studien. Da es keine randomisierten Studien zu SARS-CoV-2 mit dieser Fragestellung gibt, stammen die Daten hauptsächlich aus InfluenzaStudien, die alle nicht während einer Pandemie durchgeführt wurden. Die Durchführung von randomisierten Studien zur Effektivität von Masken während der SARS-CoV-2-Pandemie wäre ethisch sicher nicht zu vertreten. Das Fehlen solcher Studien als fehlende Evidenz zu werten wird der Situation jedoch nicht gerecht. Seit der Publikation des Positionspapiers [2] sind weitere Arbeiten erschienen, die wir hier kurz darstellen möchten: Dr. Yuen Kwok-Yung aus Hongkong, immerhin der Entdecker des SARS-Virus im Jahre 2003, konnte im Tierexperiment mit dem SARS-CoV-2-Virus eine absolute Risikoreduktion in Bezug auf Infektionen von $50 \%$ feststellen (numbers needed to treat $[N N T]=2$ ), wenn die Tiere im Käfig mittels einer Maske getrennt wurden [20]. Wichtiger aber noch, weil praxisnäher sind Daten aus der aktuellen Pandemie. Vergleiche von Populationen/ Ländern, die zu unterschiedlichen Zeitpunkten die Maskenpflicht eingeführt haben, legen dabei die Effektivität auch der Community-Masken nahe [21]. Auch die kürzlich vorgelegte Studie des IZA, die die Entwicklung der Infektionszahlen aus Jena nach der Einführung der Maskenpflicht mit Kontrollkollektiven verglich, zeigt eine eindeutige Effektivität der Masken für den Infektionsschutz in der Bevölkerung (http://ftp.iza.org/ dp13319.pdf). Eine gerade veröffentlichte Studie geht sogar davon aus, dass in Italien zwischen dem 6. April und dem 9. Mai 78000 und in New York zwischen dem 17. April und dem 9. Mai 66000 Infektionen alleine durch das Tragen von Masken verhindert wurden [16]. 
Die Effektivität der Masken hängt im Wesentlichen von den folgenden 3 Faktoren ab:

1. Wie hoch ist die Selbstschutzfunktion der Maske? (Diese kann theoretisch sogar negativ sein für den Fall, dass es durch die Verschmutzung der Maske zu einer erhöhten Infektionsrate beim Träger kommt.)

2. Wie hoch ist die Fremdschutzfunktion der Maske?

3. Wie hoch ist die Adherence in der Bevölkerung?

Eine mathematische Modellierung hierzu wurde im Juni 2020 veröffentlicht [22]. Die Autoren stellen in Ihrer Arbeit 2 mathematische Modelle vor. Ein sog. „Branching Model=Verzweigungsmodell“, welches die Ausbreitung des Coronavirus unter Modellierung der $3 \mathrm{og}$. Faktoren berechnet, und ein Kompartment Modell, bei dem die zugrunde liegende Kohorte (Bevölkerung) in Maskenträger und Nicht-Maskenträger unterteilt wird. Mit dem letztgenannten Modell lässt sich vor allem die Fremd- und Eigenschutzkomponente herausarbeiten. Die Autoren kommen zum Schluss, dass sogar bei einem gestiegenen Infektionsrisiko durch Maskenverschmutzung für den Träger der Nettoeffekt für die Gesamtbevölkerung immer noch positiv wäre. Anhand des Branching Models lässt sich dabei ablesen, wie sich der Reproduktionsindex (R) in Abhängigkeit der 3 o.g. Faktoren entwickelt.

Als 1976 in Deutschland die Gurtpflicht eingeführt wurde, war der Aufschrei zunächst groß. Man fühlte sich bevormundet und in seiner persönlichen Freiheit eingeschränkt. Heutzutage steigt man dagegen nicht mehr gerne in ein Auto ein, wenn der Airbag fehlt, vom Sicherheitsgurt ganz zu schweigen. Bei der Maskenpflicht im Rahmen der Coronapandemie ist es etwas komplizierter, da hier nicht der Selbstschutz, sondern vor allem der Fremdschutz im Vordergrund steht. Es braucht daher momentan vor allem Folgendes: Ärzte, die sinnvolle Maßnahmen der Regierung unterstützen und mit gutem Beispiel vorangehen. Viel wichtiger ist aber die Stärkung des Al- truismus in der Gesellschaft. Das Virus wird uns zeigen, ob unsere Gesellschaft dazu bereit und in der Lage ist.

\section{Interessenkonflikt}

Der Autorinnen/Autoren geben an, dass kein Interessenkonflikt besteht.

\section{Autorinnen/Autoren}

\section{PD Dr. Dominic Dellweg}

Prof. Dr. Michael Pfeifer

Klinik und Poliklinik für Innere Medizin II, Universitätsklinikum Regensburg Abteilung für Pneumologie, Fachklinik für Lungenerkrankungen Donaustauf Krankenhaus Barmherzige Brüder, Klinik für Pneumologie und konservative Intensivmedizin, Regensburg

Michael.Pfeifer@klinik.uni-regensburg.de

\section{Korrespondenzadresse}

PD Dr. Dominic Dellweg

Fachkrankenhaus Kloster Grafschaft GmbH

Akademisches Lehrkrankenhaus der

Philipps-Universität Marburg

Annostr. 1

57392 Schmallenberg Grafschaft

E-Mail: d.dellweg@fkkg.de

\section{References}

[1] Callewaert C, Van Nevel S, Kerckhof FM et al. Bacterial Exchange in Household Washing Machines. Front Microbiol 2015; 6: 1381

[2] Dellweg D, Lepper PM, Nowak D et al. Position Paper of the German Respiratory Society (DGP) on the Impact of Community Masks on Self-Protection and Protection of Others in Regard to Aerogen Transmitted Diseases. Pneumologie 2020; 74: 331336. doi:10.1055/a-1175-8578

[3] Wilson NM, Norton A, Young FP et al. Airborne transmission of severe acute respiratory syndrome coronavirus- 2 to healthcare workers: a narrative review. Anaesthesia 2020. doi:10.1111/anae. 15093

[4] Schwarz K, Biller H, Windt $\mathrm{H}$ et al. Characterization of exhaled particles from the healthy human lung - a systematic analysis in relation to pulmonary function variables. J Aerosol Med Pulm Drug Deliv 2010; 23: 371-379

[5] Schwarz K, Biller H, Windt $\mathrm{H}$ et al. Characterization of exhaled particles from the human lungs in airway obstruction. J Aerosol Med Pulm Drug Deliv 2015; 28: 52-58
[6] Chao CYH, Wan MP, Morawska L et al. Characterization of expiration air jets and droplet size distributions immediately at the mouth opening. J Aerosol Sci 2009; 40: 122-133

[7] Fowler RA, Guest CB, Lapinsky SE et al. Transmission of severe acute respiratory syndrome during intubation and mechanical ventilation. Am J Respir Crit Care Med 2004; 169: 1198-1202

[8] Anderson EL, Turnham P, Griffin JR et al. Consideration of the Aerosol Transmission for COVID-19 and Public Health. Risk Anal 2020; 40: 902-907

[9] Bahl P, Doolan C, de Silva C et al. Airborne or droplet precautions for health workers treating COVID-19? J Infect Dis 2020. doi:10.1093/infdis/jiaa189

[10] Buonanno G, Stabile L, Morawska L. Estimation of airborne viral emission: Quanta emission rate of SARS-CoV-2 for infection risk assessment. Environ Int 2020; 141: 105794

[11] Correia G, Rodrigues L, Gameiro da Silva M et al. Airborne route and bad use of ventilation systems as non-negligible factors in SARS-CoV-2 transmission. Med Hypotheses 2020; 141: 109781

[12] Eissenberg T, Kanj SS, Shihadeh AL. Treat COVID-19 as Though It Is Airborne: It May Be. AANA J 2020; 88: 29-30

[13] Morawska L, Cao J. Airborne transmission of SARS-CoV-2: The world should face the reality. Environ Int 2020; 139: 105730

[14] Setti L, Passarini F, De Gennaro G et al. Airborne Transmission Route of COVID-19: Why 2 Meters/6 Feet of Inter-Personal Distance Could Not Be Enough. Int J Environ Res Public Health 2020. doi:10.3390/ ijerph17082932

[15] Stadnytskyi V, Bax CE, Bax A et al. The airborne lifetime of small speech droplets and their potential importance in SARS-CoV-2 transmission. Proc Natl Acad Sci U S A 2020; 117: 11875-11877

[16] Zhang R, Li Y, Zhang AL et al. Identifying airborne transmission as the dominant route for the spread of COVID-19. Proc Natl Acad Sci U S A 2020. doi:10.1073/ pnas.2009637117

[17] van Doremalen N, Bushmaker T, Morris DH et al. Aerosol and Surface Stability of SARSCoV-2 as Compared with SARS-CoV-1. N Engl J Med 2020; 382: 1564-1567

[18] Chia PY, Coleman KK, Tan YK et al. Detection of air and surface contamination by SARS-CoV-2 in hospital rooms of infected patients. Nat Commun 2020; 11: 2800

[19] Jefferson T, Del Mar CB, Dooley L et al. Physical interventions to interrupt or reduce the spread of respiratory viruses. Cochrane Database Syst Rev 2011; 7: CD006207

[20] Chan JF, Yuan S, Zhang AJ et al. Surgical mask partition reduces the risk of noncontact transmission in a golden Syrian hamster model for Coronavirus Disease 2019 (COVID-19). Clin Infect Dis 2020. doi:10.1093/cid/ciaa644 
[21] Cheng VC, Wong SC, Chuang VW et al. The role of community-wide wearing of face mask for control of coronavirus disease 2019 (COVID-19) epidemic due to SARSCoV-2. J Infect 2020; 81: 107-114. doi:10.1016/j.jinf.2020.04.024

[22] Stutt ROJH, Retkute R, Bradley M et al. A modelling framework to assess the likely effectiveness of facemasks in combination with 'lock-down' in managing the COVID-

19 pandemic. Proc R Soc A 2020.

doi:10.1098/rspa.2020.0376

\section{Publikationshinweis}

Leserbriefe stellen die Meinung des Autors dar und entsprechen nicht unbedingt der Meinung von Herausgebern oder Verlag. Herausgeber und Verlag behalten sich vor, Leserbriefe nicht, gekürzt oder in Auszügen zu veröffentlichen.
Bibliografie

DOI https://doi.org/10.1055/a-1199-4557

Pneumologie 2020; 74: 409-411

(c) Georg Thieme Verlag KG

Stuttgart · New York

ISSN 0934-8387 